\title{
Reparación de hipospadias mediante la Técnica de Snodgrass
}

\author{
Serrano Durbá A, Pacheco Bru JJ, Domínguez Hinarejos C, Estornell Moragues F, \\ Nome C, Martínez Verduch M, García Ibarra F. \\ Unidad de Urología Infantil. Hospital Infantil La Fe. Valencia.
}

Actas Urol Esp. 2007;31(5):528-531

\section{RESUMEN}

REPARACIÓN DE HIPOSPADIAS MEDIANTE LA TÉCNICA DE SNODGRASS.

Estudio retrospectivo de 124 pacientes (edad media: 3,8 años) con hipospadias peneano medio: $48,3 \%$ (60 niños), peneano distal: 45,9\% (57) y coronal 5,6\% (7), de los cuales el $25,8 \%$ (16) presentaban incurvación ventral y el 4,8\% (6) procedian del fracaso de otra técnica. Todos ellos intervenidos según técnica de Snodgrass, retirando la sonda entre el 6 y $7^{\circ}$ día en la mayoría. La tasa global de complicaciones fue del 12\%: 9 fístulas $(7,2 \%)$ y 6 estenosis de meato $(4,8 \%)$. El resultado estético fue satisfactorio en todos los casos, quedando incluso el glande cubierto por prepucio el 57,3\%.

Palabras clave: Hipospadias. Uretroplastia. Niños.

\section{ABSTRACT}

HYPOSPADIAS REPAIR WITH SNODGRASS TECHNIQUE

Retrospective study of 124 patients (average age: 3.8 years) with midpenile hypospadias: 48,3\% (60 children), distal penile: 45,9\% (57) and coronal 5,6\% (7), of which the $25.8 \%$ (16) presented ventral curvature and the $4,8 \%$ (6) resulting from the complication of another previous technique. All of them were operated according to Snodgrass ${ }^{-}$technique, removing the catheter between the $6^{\text {th }}$ and $7^{\text {th }}$ day in most of them. The global rate of complications was of $12 \%$ : 9 fistulae $(7,2 \%)$ and 6 meatal stenosis $(4,8 \%)$. Aesthetic result was satisfactory in all cases, getting glans covered by foreskin in $57,3 \%$.

Keywords: Hypospadias. Urethroplasty. Children.

$\mathrm{E}^{\prime}$ tratamiento quirúrgico del hipospadias continúa siendo uno de los aspectos más controvertidos dentro de la urología infantil. Existen numerosas técnicas, sin ser ninguna de ellas de eficacia claramente superior. En la última década han aparecido nuevas técnicas, o se han modificado algunos procedimientos ya existentes. Todas ellas tienen como objetivos:

a) conseguir un pene recto,

b) con una uretra de calibre normal que se extienda hasta el ápex,

c) obteniendo un glande y un meato lo más estético posible ${ }^{1-3}$.

La técnica de uretroplastia mediante incisión y tubularización de la placa uretral (TIP) fue descrita en 1994 por Warren T. Snodgrass para la corrección de hipospadias distales ${ }^{4}$, siendo una modificación de la cirugia original descrita por Thiersch-Duplay en 1869. Consiste en la incisión relajante de la placa uretral en su línea media, aumentando el ancho de la placa y permitiendo su tubularización sin tensión. La técnica de Snodgrass ha tenido una gran aceptación, en gran parte debido a su gran versatilidad, sencillez y buenos resultados estéticos, siendo preconizada por algunos autores como sustitutiva de las técnicas de Onlay, Mathieu y Duckett ${ }^{5,6}$.

\section{MATERIAL Y MÉTODOS}

Hemos realizado un estudio retrospectivo de 124 pacientes sometidos a la técnica de Snodgrass (febrero de 2001 a diciembre de 2005) en 
nuestro servicio. La edad media en el momento de la intervención era de 3,8 años $(1,3-14,1)$.

La localización preoperatoria del meato uretral fue: 7 coronal o en surco balano-prepucial $(5,6 \%)$, 57 peneano distal $(45,9 \%), 60$ peneano medio (48,3\%). El 25,8\% (16) de los pacientes presentaban una incurvación asociada y el 4,8\% (6) había sido intervenido previamente con otra técnica de reconstrucción (Tabla 1).

Tabla 1

Características clínicas preoperatorias.

\begin{tabular}{lc}
\hline Caracteristicas clinicas & $\begin{array}{c}\text { No pacientes } \\
\text { n (\%) }\end{array}$ \\
\hline Localización meato & \\
$\quad$ Coronal & $7(5,6)$ \\
Peneano distal & $57(45,9)$ \\
Peneano medio & $60(48,3)$ \\
Total & 124 \\
Incurvación previa & $16(25,8)$ \\
Cirugía previa & $6(4,8)$ \\
MAGPI & 1 \\
ONLAY & 2 \\
Uretroplastia* & 2 \\
2+3 & 1 \\
\hline
\end{tabular}

*algún tipo de uretroplastia realizada en otro centro)

\section{RESULTADOS}

\section{Resultados operatorios}

En 100 pacientes $(80,6 \%)$ se realizó únicamente la uretroplastia, no siendo necesaria ninguna actuación adicional para la corrección de la incurvación peneana, sin embargo en 24 de los 32 pacientes con incurvación fue necesaria la desincurvación, realizándose en 7 una plicatura tipo Nesbitt y en 17 la resección de la corda. En todos los pacientes se conformó la neouretra utilizando una sutura continua de material reabsorbible como el ácido Poliglicólico (Dexon) o polidioxanona monofilamento (PDS) de 6/0 (90\%) o 7/0 (10\%). El colgajo desepitalizado procedía en 99 pacientes $(79.8 \%)$ del prepucio, realizándose una transposición lateral izquierda en 73 (73,73\%) pacientes, lateral derecha en $17(17,17 \%)$ pacientes y una trasposición medial mediante perforación del colgajo en 10 pacientes $(10,10 \%)$. En los pacientes donde no se pudo obtener un colgajo prepucial valido, este se obtuvo de la cara ventral del pene en 18 pacientes $(14,5 \%)$ o del escroto en 1 pacientes $(0,8 \%)$ realizándose un ascenso medial del colgajo en ambos casos, mientras que en 6 pacientes realizamos espongioplastia sin colgajo.

\section{Cuidados postoperatorios}

En 112 pacientes (90.3\%) se colocó como derivación urinaria una sonda de Nélaton multiperforada, en $1(0,8 \%)$ una cistostomía suprapúbica y en $11(8,8 \%)$ una sonda uretral tipo Foley. En 81 pacientes $(65,3 \%)$ el calibre del tutor utilizado era $8 \mathrm{Ch}$, en 12 pacientes $(9,6 \%) 6 \mathrm{Ch}, 24$ pacientes $(19,3 \%) 10$ Ch y solamente en 7 (5,6\%) se utilizó una sonda de calibre $12 \mathrm{Ch}$. La sonda se retiró al

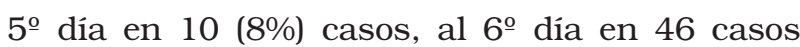
(37\%), al $7^{\circ}$ día en $58(46,7 \%)$ y en $10(8 \%)$ al $8^{\circ}$ día. Se colocó vendaje en 99 pacientes $(79,8 \%)$ y no se vendó en 25 pacientes $(20,2 \%)$, siendo retirado en apósito el $1^{\mathrm{er}}$ día en 7 casos $(7 \%)$, el $2^{\circ}$ día en 71 casos $(71,7 \%)$, el $3^{\circ}$ día en 18 casos $(18,1 \%)$ y el $4^{\circ}$ día en 3 casos (3\%). La estancia media fue de 6,6 días (rango 5-9), siendo dado de alta el $5^{\circ}$ día 1 paciente $(0,85)$, el $6^{\circ}$ día 43 pacientes $(34,6 \%)$, el $7^{\circ}$ día 63 pacientes $(50,8 \%)$, el $8^{\underline{a}}$ día 16 pacientes $(13,7 \%)$ y 1 el $9^{\circ}$ día $(0,8 \%)$.

\section{Resultados estéticos}

$\mathrm{El}$ resultado estético final fue satisfactorio en todos los pacientes, presentando todos un meato en ápex de configuración vertical, con un glande cubierto en 53 pacientes $(42,7 \%)$ y descubierto en 71 pacientes $(57,3 \%)$.

\section{Complicaciones}

Durante el postoperatorio precoz, 108 pacientes (87\%) no tuvieron ningún tipo de incidencia durante su ingreso, 14 pacientes $(9,6 \%)$ presentaron edema importante que precisó la aplicación de corticoides tópicos, 2 pacientes $(1,6 \%)$ desarrollaron un hematoma de resolución espontánea, 1 paciente $(0,8 \%)$ sufrió salida de la sonda de forma accidental y otro presentó una epidimitis aguda.

Se obtuvo un calibre meático adecuado en 102 pacientes $(82,2 \%), 13$ pacientes $(10,4 \%)$ presentaban un meato ligeramente estenótico que no precisó intervención quirúrgica para su corrección y 6 pacientes $(4,8 \%)$ presentaron estenosis severas subsidiarias de reintervención quirúrgica (meatotomía). La fístula de la neouretra fue la $1^{\underline{a}}$ complicación en frecuencia, 9 casos $(7,2 \%)$, de los 
cuales en 2 estaba asociada a una estenosis severa de meato. De ellos, 7 fueron reintervenidos, 5 meatotomías aisladas y 2 meatotomías con fistulorrafia (Tabla 2).

Tabla 2

Resultados estéticos y complicaciones.

\begin{tabular}{lc}
\hline Caracteristicas clinicas & $\begin{array}{c}\text { No pacientes } \\
\text { n (\%) }\end{array}$ \\
\hline $\begin{array}{l}\text { Resultado estético } \\
\quad \text { Satisfactorio } \\
\quad \text { Insatisfactorio } \\
\quad \text { Meato en ápex }\end{array}$ & $124(100)$ \\
Configuración Meato & 0 \\
$\quad$ Vertical & $124(100)$ \\
$\quad$ Transversal/circular & $124(100)$ \\
Glande cubierto & 0 \\
Complicaciones & $53(42,7)$ \\
$\quad$ Estenosis meato & \\
$\quad$ Fistulas & $6(4,8)$ \\
Dehiscencia rafia & $9(7,2)$ \\
$\quad$ Tasa global & 0 \\
* nota: la tasa global por pacientes es $13(10,4 \%)$ & $15(12) *$ \\
pacientes presentaban de forma sincrónica una estenosis de \\
meato más una fistula uretral.
\end{tabular}

\section{DISCUSIÓN}

Desde su publicación en 1994, la uretroplastia de Snodgrass ha adquirido una notable popularidad en un corto periodo de tiempo, muestra de ello, Franzoni y Decter ${ }^{7}$ reportan en 1997 (3 años después de publicarse la técnica) la utilización de esta técnica en el $82 \%$ de los pacientes con hipospadias distales intervenidos en su centro. En nuestro servicio, desde su introducción en febrero del 2001, el Snodgrass ha adquirido un papel preponderante en el tratamiento de hipospadias peneanos medios y distales, desplazando casi por completo al resto de técnicas utilizadas con anterioridad para hipospadias en la misma localización. Las causas de esta gran popularidad son diversas, por un lado su sencillez técnica, que reduce la curva de aprendizaje de forma significativa ${ }^{8}$ y por otro lado, su gran versatilidad y excelentes resultados cosméticos.

Uno de los conceptos claves en el TIP es la preservación de la placa uretral. Generalmente, se acepta que la tasa de complicaciones es mayor en las técnicas que no preservan la placa uretral ${ }^{3}$, apreciando en los últimos 15 años una tendencia a usar técnicas que incorporan la placa a la neou- retra. Algunos autores han defendido que la placa uretral no es un tejido fibroso, sino que esta formada por tejidos sanos bien vascularizados que influirían en la rápida reepitelización de la neouretra sin ocasionar una fibrosis importante. En este sentido, Baskin et $\mathrm{al}^{9}$ demostraron en estudios histológicos de penes fetales con hipospadias distales, la existencia de amplios senos vasculares por debajo de la placa uretral.

Una ventaja importante del Snodgrass sobre otras técnicas de reconstrucción (como el Onlay, Mathieu, etc.) es el resultado cosmético final, con la creación de un neomeato de configuración vertical, que le da al glande una apariencia casi normal. Nuestra experiencia concuerda plenamente con los trabajos publicados, obteniendo unos resultados estéticos plenamente satisfactorios, ya que en todos los casos obtuvimos un meato localizado en ápex, de orientación vertical y en la mitad de los pacientes un glande recubierto por piel prepucial.

El Snodgrass desde su descripción original para el tratamiento de hipospadias distales, se ha mostrado como una técnica muy versátil. Este hecho se ha puesto de manifiesto con la progresiva ampliación de sus indicaciones. Muchos autores han elogiado los buenos resultados de la técnica de Snodgrass en reintervenciones de pacientes con correcciones de hipospadias fallida ${ }^{6,10,11}$. La técnica de Snodgrass es ideal para la reparación tras fracaso del Mathieu, Magpi y Onlay, debido a que, teóricamente, la placa uretral permanece con una buena vascularización sin haber sido agredida por la cirugía previa. Sin embargo, no es aplicable cuando se ha realizado una técnica de Duckett o de Thiersch-Duplay, en las que la placa uretral no permanece intacta ${ }^{12}$. La técnica no difiere significativamente de la descrita para la reparación primaria del hipospadias. En nuestra experiencia, 4 pacientes, el Snodgrass nos permite reintervenir hipospadias con las máximas garantías de éxito, incluso cuando no disponemos de piel prepucial. Otra de las aplicaciones descritas en los últimos años es la reparación de hipospadias proximales, siempre que no exista una curvatura ventral severa, con buenos resultados cosméticos y un índice de complicaciones similar al presentado por el resto de técnicas ${ }^{5}$, en este sentido nuestra experiencia es nula prefiriendo aplicar en estos casos las técnicas de Koyanagi o de Hayashi. 
Las dos contraindicaciones para la realización de la técnica de Snodgrass son la presencia de una placa uretral angosta o hipoplásica que haga imposible la tubulización de la uretra o la existencia de una corda ventral severa que obligue a seccionar la uretra. En nuestra casuística, 32 pacientes, la incurvación no fue un impedimento para la realización de la técnica de Snodgrass, siempre que previa a la uretroplastia se realice la ortoplastia, bien mediante una plicatura tipo Nesbitt o bien mediante la resección de la corda.

En 1996 Snodgrass publicó los resultados del tratamiento de 328 pacientes con hipospadias distales, en los que obtuvo un indice de complicaciones global del 7\%13. En 1998 Snodgrass y col. publicaron la primera serie de uretroplastias TIP en hipospadias más severos, obteniendo una tasa de complicaciones del $11 \%{ }^{14}$, resultados similares a los obtenidos en nuestra casuística (tasa global complicaciones 12\%). Cuando se analizan los resultados acumulados de las diversas series publicadas ${ }^{15}$ se aprecia que la complicación más habitual es la formación de fístulas (4\%), seguido de la estenosis de meato (3\%) y la dehiscencia de la rafia (1\%). En nuestra experiencia, las 2 complicaciones más frecuentes son la fístula (en casi todos los casos asociada a estenosis), y la estenosis de meato siendo nuestros resultados equiparables a los publicados en la literatura. A nuestro entender, con el fin de minimizar las estenosis de meato, es fundamental no forzar el ascenso de la sutura en la uretroplastia en el ápex glandar para evitar el cierre excesivo de la placa distal, aspecto en el que coincidimos con otros autores ${ }^{8}$ aunque con ello se corre el riesgo de dejar el meato con un aspecto desprendido. Borer ${ }^{10}$ en un estudio realizado en 520 pacientes no observa diferencias significativas en el indice de complicaciones entre las técnicas de Mathieu, Onlay y Snodgrass.

\section{CONCLUSIÓN}

En nuestra experiencia, la uretroplastia utilizando la técnica de Snodgrass es una intervención muy versátil que puede aplicarse en una amplia gama de trastornos hipospádicos, desde hipospadias peneanos distales y medios hasta su utilización en hipospadias proximales y en reintervenciones con placa uretral intacta. Aporta excelentes resultados estéticos, con la conformación de un meato de orientación vertical, presentando una baja tasa de complicaciones.

\section{REFERENCIAS}

1. Duckett JW. Hypospadias. In Walsh PC, Retik AB, Stamey TA, Vaughan ED (Eds): Campbell's Urology ed 7, vol 2. 1998; p. 2093.

2. García-Ibarra F, Martínez-Verduch M. Tratamiento del hipospadias. En: Asociación Española de Urología, editor. Hipospadias masculina. Estado actual del tratamiento. Tema monográfico del LIV Congreso Nacional de Urología. Madrid. ENE Ediciones; 1989;p 95.

3. Ghali AM., El-Malik EM. Ibrahim AH One-Stage Hipospadias Repair. Experience with 544 cases. Eur Urol.1999; 36(5):436-442.

4. Snodgrass W. Tubularized incised plate urethroplasty for distal hypospadias. J Urol 1994; 151(2):464-465.

5. Snodgrass W, Lorenzo A. Tubularized incised-plate urethroplasty for proximal hypospadias. BJU International 2002; 89(1):90-93.

6. Snodgrass W, Lorenzo A. Tubularized incised-plate urethroplasty for hypospadias reoperation. B.J.U. International 2002; 89(1):98-100.

7. Franzoni DF, Decter RM. Distal hypospadias repair by the modified Thiersch-Duplay technique with or without hinging of the urethral plate: a near ideal way to correct distal hypospadias. AAP. San Francisco 1998.

8. Castellán M, Labbie A, Lince L, Gosalbez R. Versatilidad de la técnica de Snodgrass para la corrección de diferentes tipos de hipospadias. Arch Esp Urol. 2000;53(7):625-628.

9. Baskin LS, Erol A, Ying WL, Cunha GR. Anatomic Studies of hypospadias. J Urol.1998;160(3):1108-1115.

10. Borer JG, Bauer SB, Peters CA, Diamond DA, Atala A, Cilento BG Jr et al. Tubularized incised plate urethroplasty: expanded use in primary and repeat surgery for hypospadias. J Urol. 2001;165(2):581-585.

11. Elbakry A. Further experience with the tubularized-incised urethral plate technique for hypospadias repair. BJU International. 2002;89(3):291-294.

12. Borer JG, Retik AB. Current trends in hypospadias repair. Urol Clin North Am 1999; 26(1): 15-37.

13. Snodgrass W, Koyle M, Manzoni G, Hurwitz R, Caldamone A, Ehrlich R. Tubularized incised plate repair: Results of a multicenter experience. J Urol 1996; 156(2):839-841.

14. Snodgrass W, Koyle M, Manzoni G, Hurwitz R, Caldamone A, Ehrlich R. Tubularized incised plate hypospadias for proximal hypospadias. J Urol 1998; 159(6):2129-2131.

15. Snodgrass W. Tubularized incised plate (TIP) hypospadias repair. Urol Clin North Am 2002; 29(2):285-290.

16. Stehr M, Lehner M Schuster T, Heinrich M, Dietz HG. Tubularized incised plate (TIP) urethroplasty (Snodgrass) in primary hypospadias repair.Eur J.Pediatr Surg 2005; 15(6):420-424.

Correspondencia autor: Dr. A. Serrano Durbá

Unidad de Urología Infantil. Hospital Universitario La Fe Avda. Campanar, 21 - 46009 Valencia. Tel.: 963862700 E-mail autor: serrano_agu@gva.es

Información artículo: Original - Urologia pediátrica

Trabajo recibido: diciembre 2006

Trabajo aceptado: febrero 2007 